\title{
EVALUACIÓN DE LA CALIDAD Y ENTONACIÓN DEL INTÉRPRETE DE SIMULTÁNEA: PAUTAS EVALUADORAS
}

\author{
Ángela Collados Aís \\ colladosais@gmail.com \\ Universidad de Granada
}

\section{Resumen}

El presente trabajo tiene como objetivo analizar las pautas evaluadoras de varios grupos diferentes de receptores de Interpretación Simultánea (IS), poniendo el foco en la entonación del intérprete. Se investiga, por una parte, si distintos receptores comparten pautas generales de evaluación de la entonación del intérprete, con independencia de su grado de monotonía. Por otra parte, se investiga si su grado de conocimiento sobre el proceso de la IS reflejaría distintas pautas y, sobre todo, una menor influencia del grado de monotonía de la entonación del intérprete sobre otros parámetros de calidad. La investigación se divide en dos estudios diferenciados en función de los sujetos y del material utilizado. Los resultados indican que, a partir de la valoración de la entonación del intérprete, es posible establecer ciertos rasgos comunes en las pautas generales de evaluación de una IS.

\begin{abstract}
"Quality assessment and intonation in simultaneous interpreting: evaluation patterns" The main goal of this paper is to analyse the evaluation patterns of various groups of Simultaneous Interpreting (SI) recipients, with a focus on interpreter intonation. The research focuses, on the one hand, on whether different recipients share general patterns in evaluating interpreter intonation, regardless of its degree of monotony. On the other hand, it looks at whether their degree of knowledge on the process of SI results in different patterns and, above all, a lower degree of influence of the interpreter's monotony as compared to other quality parameters. The research is composed of two studies based on different subjects and materials used. Results point at some common traits in the evaluation patterns of SI, starting from the evaluation of the interpreter's intonation.
\end{abstract}


Palabras clave: Interpretación Simultánea. Evaluación de la calidad. Parámetros de calidad. Entonación. Pautas evaluadoras.

Keywords: Simultaneous interpreter. Quality evaluation. Quality parameters. Intonation. Evaluation patterns.

Manuscript received on April 19, 2015 and accepted for publication on September 19, 2015. 


\section{Planteamiento general}

En el presente trabajo se expone una investigación que tiene como objeto analizar las pautas evaluadoras de los receptores de una Interpretación Simultánea (IS) poniendo el foco en la entonación del intérprete. En este sentido nos interesa, por una parte, investigar si existen por parte de distintos tipos de receptores pautas generales de evaluación -entendidas como comportamientos estables de evaluación- de la entonación del intérprete, con independencia de su grado de monotonía. Se analiza de este modo el lugar que ocupa la entonación del intérprete en el engranaje de las variables sometidas a evaluación: la valoración general de la IS, la evaluación del parámetro de calidad por excelencia de la IS (la transmisión correcta del sentido), así como de los juicios que los receptores hacen sobre la actitud -entendida como la disposición del intérprete frente a la propia tarea de interpretar-, la profesionalidad y la fiabilidad del intérprete. Por otra, se pretende analizar si también existen pautas de evaluación de la entonación que, según su grado de monotonía, impliquen vinculaciones con las evaluaciones de las demás variables anteriormente descritas según el grado de conocimiento sobre el proceso de la interpretación que posean los receptores.

La investigación parte de dos estudios diferenciados en cuanto a los sujetos y al material utilizado. En el primer estudio, se contemplan sujetos con un grado bajo y medio de conocimiento sobre el proceso de la IS, respectivamente, y se incluye la evaluación de cuatro IS con distintos grados de monotonía. El segundo estudio contempla las evaluaciones de seis sujetos con un alto grado de conocimiento sobre el proceso de la IS, de un corpus de 30 IS grabadas del Parlamento Europeo a través del canal Europe by Satellite (EbS). Ambos estudios forman parte de una investigación más amplia que contempla, por una parte, la realización de distintos grupos de discusión y, por otra, el análisis cualitativo de un corpus auténtico de IS.

El detonante de la presente investigación fueron los resultados de otra investigación sobre cómo afectaba el orden de exposición a una IS con entonación monótona sobre otros parámetros de calidad según su posición frente a una IS con entonación no monótona (Collados Aís 2008). Los resultados, 
si bien establecían que la entonación monótona era más castigada si ocupaba el lugar inmediatamente posterior a la escucha de una IS con entonación no monótona, arrojaban márgenes de diferencias prácticamente idénticos si esta ocupaba el primer lugar de escucha y era seguida por una IS con entonación no monótona. La IS con entonación no monótona era correlativamente premiada más o menos según el orden de escucha que ocupara, al igual que la IS con entonación monótona se adaptaba automáticamente por parte de los usuarios en la intensidad de su "castigo".

\section{Estado de la cuestión}

\subsection{Estudios de evaluación de la calidad y entonación en Interpretación} Simultánea

Desde los inicios de los estudios de la interpretación se ha venido destacando la importancia que reviste una buena presentación para la calidad de una interpretación. En este sentido, se ha resaltado la trascendencia de la voz del intérprete, que no ha de ser ni desagradable ni monótona (Katz 1989), debiendo este profesional hacer uso de los medios prosódicos que tiene a su alcance (Déjean Le Féal 1981). En el ámbito de las asociaciones profesionales tampoco se ha obviado la relevancia de aspectos tales como la voz o la entonación (véase, entre otros, AIIC 2004). A pesar de ello, los aspectos vocales no verbales empezaron a estudiarse después de que ya se hubiese desarrollado la investigación sobre los aspectos verbales. La justificación puede estar en el propio desarrollo tardío de estudios específicos y de técnicas e instrumentales adecuados para la medición de este tipo de parámetros, ya que incluso en el ámbito de la psicología y la lingüística los primeros estudios se centraron en los parámetros verbales (cf. Barrango-Droege, Collados Aís \& Pazos Bretaña 2011).

En 1994, Shlesinger publica un trabajo que tiene la voz como objeto de estudio en la investigación. La autora comparó la emisión vocal de la IS con la de la lectura, concluyendo que la entonación del intérprete podía ser calificada de sui géneris ya que existirían rasgos peculiares propios de la entonación y, en general, de su emisión vocal. Estas características se centran fundamentalmente en un incremento importante de pausas no gramaticales en posiciones "no usuales" y en una prosodia específica con acentuación de elementos que en el habla espontánea o leída no se producirían.

Ahrens (2005) aporta un estudio exhaustivo sobre la entonación basada en un corpus de IS inglés-alemán. Sus conclusiones apuntan también a aspectos específicos del estilo discursivo del intérprete condicionados por el propio 
proceso de la IS y las circunstancias comunicativas específicas en las que se produce (Ahrens 2005: 230). El intérprete tiende a una segmentación prosódica mayor del texto por motivos estratégicos (Ahrens 2005: 227). Por su parte, Nafá (2005), en una amplia investigación dedicada íntegramente a la entonación a partir de un corpus extraído del Parlamento Europeo, muestra el uso de estrategias de entonación y retóricas a las que recurren los intérpretes para la comunicación en su ejercicio profesional, y que se centran en la organización y estructuración de sus discursos. Estas estrategias no se aplican en todos los casos ni durante toda una interpretación, posiblemente porque esta entonación comunicativamente aceptable se ve alterada por las demandas cognitivas del proceso de la IS (Nafá 2005: 678).

Por lo que respecta a los estudios de expectativas de calidad de la interpretación en distintos grupos de usuarios -provenientes de distintos ámbitos o especialidades y con independencia de otros factores como el género o la edad-, así como en los propios intérpretes, estos han venido mostrando una incidencia claramente menor de los parámetros no verbales frente a los parámetros verbales, tales como la transmisión correcta y la cohesión (cf. Collados Aís et al. 2011). Únicamente en trabajos en los que el foro en el que tienen lugar -cine y televisión- determinan unas características específicas de los sujetos (Kurz \& Pöchhacker 1995, Russo 2005), los parámetros no verbales adquieren una posición algo más relevante que en los anteriores, aunque sin modificar el ranking habitual de preferencias.

Sin embargo, en un experimento sobre evaluación de la calidad realizado por Collados Aís en 1998 no se confirmaron estas expectativas en cuanto a la entonación monótona de la IS. Por el contrario, se constató una incidencia alta de la entonación monótona no solo sobre la evaluación global de trabajo del intérprete, sino también sobre los demás parámetros de calidad -verbales y no verbales- barajados en la investigación y sobre aspectos tales como la fiabilidad o la profesionalidad del intérprete. Estos resultados denotarían la influencia, incluso no consciente, de los elementos vocales no verbales sobre los juicios emitidos por los oyentes sobre el propio intérprete, su personalidad y credibilidad, y refrendarían en los estudios de interpretación los datos de las investigaciones llevadas a cabo en el ámbito de la psicología (cf. Collados Aís 1998); algo que se ha confirmado en experimentos posteriores sobre otros aspectos no verbales como las primeras impresiones que se forman los sujetos sobre el intérprete de simultánea (cf. García Becerra 2012). Si bien, en el caso concreto de la entonación, la replicación posterior del experimento no corroboró estos resultados, sí puso de relieve un dato interesante: la entonación no fue bien valorada tampoco en el resto de interpretaciones (interpretación 
control e interpretaciones manipuladas de otros diez parámetros de calidad) sometidas a evaluación (Collados Aís 2007). Tras revisar dos experimentos anteriores que incluyeron la misma interpretación control, se comprobó que el parámetro entonación fue el peor valorado del total de once parámetros de calidad sometidos a evaluación (Collados Aís 1998; Pradas Macías 2003). Estudios anteriores (Garzone 2003) y posteriores (Holub 2010) sí ratificaron esta incidencia. Se comprobó, además, que la influencia de la entonación monótona sobre la evaluación sería también diferente según el tipo de usuario, a pesar de que la hipótesis de partida no preveía estos resultados dado que se consideraba que su influencia debía de ser menor que en el caso de las expectativas. De esta forma se comprobó que, por ejemplo, los sujetos provenientes del ámbito de ciencias serían menos sensibles a las características de la entonación del intérprete que los de humanidades, no solo en sus expectativas, sino también en su evaluación de una interpretación concreta (Collados Aís 2010). No obstante, la reciente revisión de estos resultados, a raíz de la preparación de este trabajo y dados los resultados descritos en el apartado siguiente, arrojó nuevamente un dato que no había sido considerado tan relevante hasta ahora: tanto en los sujetos de ciencias como en los de humanidades el patrón de evaluación revela que todos los parámetros sometidos a evaluación son mejor valorados que la entonación y que las diferencias son más acusadas en el caso de los sujetos de ciencias. Además, se constata que son precisamente los parámetros verbales sometidos a evaluación (transmisión correcta y cohesión), así como la valoración global de la interpretación, los que menores diferencias arrojan entre los dos grupos de sujetos, frente a la agradabilidad de la voz o la valoración de la actitud del intérprete (cf. Collados Aís 2010). Otros estudios han sido realizados siguiendo metodologías provenientes de las ciencias sociales y con un carácter más cualitativo (grupos de discusión). Los resultados de estos estudios indican que los usuarios partirían efectivamente de un cierto grado de monotonía del intérprete en su modelo o estereotipo de interpretación (Collados Aís 2009). Este dato podría matizar los resultados de los distintos trabajos efectuados sobre la entonación del intérprete y subrayaría la necesidad de partir de este modelo para interpretar los posibles resultados que se obtengan en cualquier estudio sobre el tema, puesto que la entonación no monótona se consideraría un elemento diferenciador, y se valoraría positivamente que un intérprete "rompiese" con ese modelo previo que incluiría cierta monotonía en la entonación. 


\subsection{Antecedente}

El antecedente inmediato de la presente investigación, o más bien el detonante, fue una investigación realizada con el objetivo de sondear la evaluación de la calidad realizada por usuarios de una interpretación monótona frente a una interpretación no monótona, así como la repercusión de contrastes entre las distintas interpretaciones que pueden recibir durante una misma sesión y sus efectos sobre la evaluación de otros parámetros de calidad (Collados Aís 2008). Dado que los resultados de las distintas investigaciones previas realizadas con el mismo material mostraban una tendencia general a la detección y castigo de la entonación monótona -pero también mostraban una cierta contradicción (véase apartado anterior)-, interesaba asimismo añadir como objetivos tangenciales datos respecto al comportamiento frente a una interpretación monótona, con independencia del orden en el que valoraban las interpretaciones.

El diseño metodológico comprendía la realización de un experimento en el que la evaluación de los sujetos se realizaba en dos grupos a fin de evaluar dos interpretaciones, una IS manipulada hacia una mayor entonación monótona (ISM) y una IS control (ISC), es decir, no manipulada en cuanto a la entonación (entonación no monótona). Por tanto, ambas interpretaciones eran idénticas, salvo por lo que respecta a la entonación. Mientras el primer grupo visualizaba y evaluaba primero la ISM y después la ISC (grupo MC), en el otro grupo los usuarios visualizaban y evaluaban primero la ISC y después la ISM (grupo CM). Participaron dieciocho sujetos, diez profesores de distintos departamentos de Filologías de la Universidad de Granada con docencia en la Facultad de Traducción e Interpretación (FTI) y ocho estudiantes de último curso de la Licenciatura de Traducción e Interpretación de la FTI, especialidad en interpretación de conferencias y lengua B inglés, repartidos en dos grupos. El material consistió en dos DVD en los que se visualiza la emisión de un discurso alemán al que se le superpone la voz de una intérprete que realiza la IS. La grabación fue precedida de estudios preliminares que la adaptaron a la realidad profesional de la interpretación y testada mediante estudios piloto, así como analizada desde el punto de vista técnico acústico y aplicada en distintas investigaciones (cf. Collados Aís 1998, 2007).

Las evaluaciones de los sujetos se recogieron en dos tipos de cuestionarios. El primero incorporaba un rango de cinco para las preguntas cerradas sobre la valoración global de la interpretación (cf. Gile 1990) y cuatro parámetros de calidad: cohesión, transmisión correcta del discurso original, agradabilidad de la voz y entonación, así como evaluaciones acerca de la profesionalidad y la fiabilidad que les produce la intérprete. En el segundo cuestionario 
se incluyeron preguntas acerca de la valoración numérica de la actitud de la intérprete (también en un rango de 5) y su definición según propuestas incluidas o añadidas por los sujetos.

En cuanto a los resultados, según vídeo visionado y posición que ocupa, todos los apartados fueron mejor valorados en la ISC por lo que respecta al grupo CM. Las mayores diferencias se dieron en el parámetro entonación, donde la ISC superó a la ISM en 3,11 puntos, así como en los apartados agradabilidad de la voz $(2,11)$ y valoración global $(1,56)$. Las menores diferencias, por el contrario, se dieron en los apartados transmisión correcta $(0,17)$ y cohesión $(0,44)$. En el caso del grupo MC, las diferencias entre las interpretaciones indicaron que la ISC superaba a la ISM en todos los apartados, salvo el destinado a transmisión correcta, en el que ambas interpretaciones obtuvieron la misma calificación (4). En el resto de apartados, las diferencias mayores se dieron en entonación $(3,33)$ y valoración global $(0,78)$. Las menores se situaron en agradabilidad de la voz $(0,23)$ y cohesión $(0,33)$, además de la valoración de la transmisión correcta. La actitud del intérprete, en el caso del grupo CM, obtuvo una puntuación de 4,11 para la ISC y un 2,88 para la ISM, lo que arrojó una diferencia de 1,23 a favor de la primera. En el caso de la profesionalidad la diferencia fue de $1,66(4,77$ frente a 3,11$)$ y en fiabilidad de 1 punto $(4,44$ frente a 3,44). En el caso del grupo MC, las valoraciones de la ISC superaron a la ISM en actitud en 2 puntos $(2,44$ frente a 4,44$)$, en fiabilidad la diferencia fue de 1,23 (3,33 frente a 4,55), y en profesionalidad de 1 punto $(3,33$ frente a 4,33). Por lo que respecta a las definiciones de dicha actitud, se produjeron diferencias tanto en la ISC como en la ISM según el orden de recepción. Por lo que respecta a la ISC, la mayor diferencia se centró en el apartado neutralidad, que obtuvo ocho menciones si ocupaba el primer lugar en el visionado, y no obtuvo ninguna mención cuando se situaba en el segundo lugar de visionado.

Al verificar la influencia de la monotonía de la IS sobre la evaluación, con independencia del orden en el que se recibía, se comprueba que la ISC obtuvo idénticas puntuaciones en las dos condiciones experimentales para los apartados de valoración global y para agradabilidad de la voz. En los demás apartados, las diferencias no superaron en ningún caso el medio punto. En el caso de la ISM, las diferencias oscilan también entre un 0,11 para los apartados cohesión (puntuación mayor en la condición experimental CM) y entonación (condición experimental MC) hasta un 0,78 en el apartado valoración global (a favor de la condición MC). De hecho, los siguientes gráficos ( 1 y 2) reflejan la inversión en los picos de valoración: 


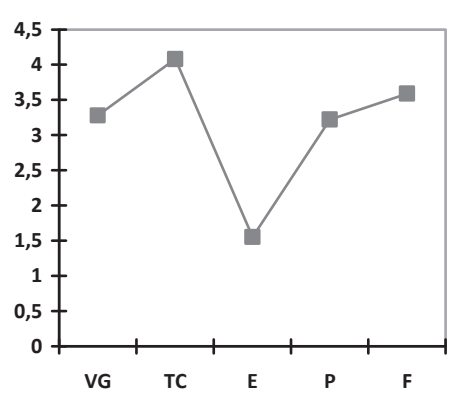

Gráfico 1. Pauta de evaluación IS monótonas

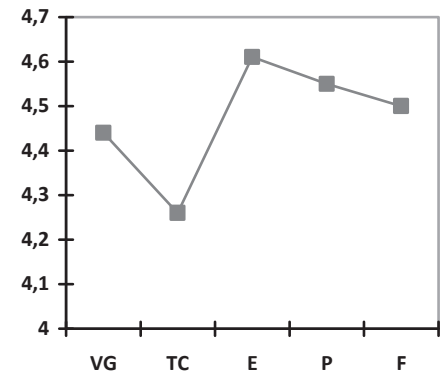

Gráfico 2. Pauta de evaluación IS no monótonas

De los resultados obtenidos y resumidos en líneas precedentes se puede deducir básicamente que a partir del reconocimiento de la monotonía de la intérprete, la ISM es valorada muy por debajo de la ISC en prácticamente todos los apartados y en cualquier condición experimental. Sin embargo, del orden de escucha de una interpretación con entonación monótona frente a una no monótona también se puede deducir que las diferencias importantes en la evaluación se producen sobre todo en las franjas en las que tenía lugar la valoración, pero no así en las propias diferencias de puntuaciones obtenidas por las interpretaciones. De este modo, se planteaban una serie de importantes interrogantes. No deja de llamar la atención, por ejemplo, que las valoraciones globales de ambas interpretaciones, y en cualquier condición experimental, se hayan movido fundamentalmente en las franjas 3 y 4 . A pesar de que la monotonía de la interpretación es detectada por los sujetos $(1,33$ y 1,44 , valoraciones respectivas según condición experimental para el parámetro entonación), el trasvase del "castigo" se ha mantenido en unos límites que parecen considerar que la ISM estaría dentro del margen de una interpretación aceptable, de calidad media, máxime cuando incluso los parámetros de contenido han sido peor valorados en la ISC (de 0,2 a 0,44 puntos). Por otra parte, la ISC, precisamente en los parámetros de contenido, ha bajado en su valoración en la condición MC, y la ISM, a pesar de que la valoración global sí decrece en un 0,78 si se trata del segundo visionado, aumenta su puntuación, aunque mínimamente, en los valores de cohesión y transmisión correcta. Respecto a la actitud, el contraste de exposición es claro, sobre todo si nos fijamos en las diferencias que se producen en la inferencia de la ISC según condición experimental. El contraste con la monotonía lleva a los sujetos a inferir en la condición MC emociones más "activas" y "positivas", como el interés o el entusiasmo cuando sin contraste percibían más la neutralidad del intérprete 
(cf. Collados Aís 1998). Sin embargo, las puntuaciones otorgadas a los distintos parámetros considerados permanecen prácticamente estables; es más, la ISC recibe un 0,44 menos en el apartado profesionalidad (valores por debajo, aunque mínimos, también los recibe en los apartados cohesión y transmisión correcta), lo que llevaría a plantear la cuestión de si realmente una mayor activación del intérprete y la vinculación de esta mayor activación con una emoción positiva, y no neutral, sería incluso considerado por los usuarios como menos profesional, en el sentido posiblemente de visualizar una mayor intervención del intérprete (cf. Kopzcynski 1994) y que esta consideración le provoque una cierta desconfianza.

\section{Estudio empírico}

\subsection{Objetivos}

Los objetivos del presente trabajo, teniendo en cuenta los resultados del estudio anterior (véase § 2.2.), se centran en analizar las evaluaciones sobre la entonación del intérprete que realizan distintos tipos de receptores de una IS a fin de:

a. Analizar globalmente el lugar que ocupa el parámetro entonación entre el resto de parámetros de calidad y variables sometidas a evaluación en una IS. En concreto, el parámetro transmisión correcta, así como la valoración global de una interpretación y los juicios sobre la actitud, la fiabilidad y la profesionalidad del intérprete.

b. Estudiar si existen pautas evaluadoras globales de la entonación, comunes a distintos receptores con diferentes grados de conocimiento previos sobre el proceso de la IS.

c. Estudiar si existen pautas evaluadoras comunes a partir de distintos grados de monotonía de la entonación entre distintos receptores con diferentes grados de conocimiento previos sobre el proceso de la IS.

\subsection{Hipótesis}

Hipótesis 1

La entonación del intérprete recibirá una valoración por sus receptores, sea cual sea su grado de monotonía y sea cual sea el grado de conocimiento de los receptores sobre el proceso de la interpretación, que se mantendrá en los límites de la franja de evaluación media/baja. 


\section{Hipótesis 2}

La entonación más monótona del intérprete influye más negativamente en la evaluación de otros parámetros de calidad en el caso de receptores de IS con un menor grado de conocimiento sobre el proceso de la interpretación.

\subsection{Variables}

La entonación del intérprete constituye la variable independiente. Como variables dependientes de estudio se consideran la transmisión correcta, la valoración general de la interpretación, la actitud, la profesionalidad y la fiabilidad del intérprete.

\subsection{Material y método}

\subsubsection{Muestra}

La muestra total de los dos estudios que se presentan es de 28 sujetos. Se contemplan tres tipos diferentes de sujetos a fin de detectar posibles regularidades y diferencias en las pautas de evaluación de la entonación del intérprete, dependiendo de su mayor o menor conocimiento acerca del proceso de una IS.

La muestra del Estudio 1 la componen 23 sujetos que evalúan, respectivamente, cuatro IS. El primer grupo de sujetos, denominado Grupo 1, está formado por cinco profesoras de la Universidad de Granada (UGR), dos de la Facultad de Psicología, especializadas en Habla, dos de Interpretación de la Facultad de Traducción e Interpretación (FTI) y una investigadora especializada en Lingüística de la Facultad de Filosofía y Letras, por una parte, y 13 sujetos, docentes de filologías de la FTI y estudiantes de último curso de la especialidad de Interpretación de la Licenciatura de Traducción e Interpretación de la UGR, por otra. La muestra del segundo grupo, denominado Grupo 2 está compuesto por cinco profesores del Departamento de Ciencia Política de la UGR, usuarios habituales de IS.

En el Estudio 2 la muestra total está compuesta por seis especialistas, investigadoras del grupo de investigación ECIS (Evaluación de la Calidad en Interpretación Simultánea) y docentes de interpretación de la UGR y de la Universidad de las Palmas de Gran Canaria, Grupo 3, que evalúan un corpus de 30 IS, respectivamente. 


\subsubsection{Materiales e instrumentos de medida}

En el Estudio 1 se han utilizado en total cuatro IS, dos interpretaciones manipuladas según su grado de monotonía (ISC e ISM) (véase § 2.2.) y dos interpretaciones no manipuladas (auténticas) del corpus que se describe a continuación: aquella que fue considerada como menos monótona (ISC') y la considerada más monótona por los sujetos del Estudio 2 (ISM'). Las valoraciones (rango de 5) fueron recogidas en un cuestionario (Anexo 1) que contemplaba los siguientes ítems en el caso del Grupo 1: valoración global, entonación y actitud. En el Grupo 2 se incluyó también la evaluación del parámetro transmisión correcta.

Respecto al Estudio 2, el material lo componen 30 IS, es decir, el número total de interpretaciones hacia el español de un corpus multilingüe de discursos en alemán, francés e inglés, grabadas de una sesión plenaria completa del Parlamento Europeo. En total, por tanto, contamos con las evaluaciones realizadas por las seis investigadoras que componen la muestra (180 evaluaciones en total). Se evaluaron las interpretaciones (rango de 5) mediante cuestionarios de evaluación idénticos a los utilizados en trabajos anteriores (cf. Collados Aís et al. 2007), aunque en el presente trabajo nos centraremos en los siguientes ítems: valoración global, entonación, transmisión correcta, profesionalidad y fiabilidad (Anexo 2)

\subsubsection{Procedimiento}

En ambos estudios los sujetos procedieron, tras la escucha de cada IS, a su evaluación mediante la cumplimentación de los respectivos cuestionarios.

\section{Resultados}

\subsection{Resultados del Estudio 1}

En cuanto a los resultados, se presentan en primer lugar los obtenidos para el Grupo 1 según interpretaciones evaluadas. Seguidamente se muestran los específicos para el Grupo 2 (incluye la valoración del parámetro transmisión correcta). Respecto a los primeros, los datos muestran que han sido detectadas las interpretaciones según su grado de monotonía y que han tenido incidencia sobre los demás ítems considerados (véanse tablas 1 a 4). En ambos casos, se toma como referencia ' $\mathrm{N}$ ', el número total de evaluaciones realizadas. 


\begin{tabular}{|l|c|c|c|c|c|}
\hline & $\mathrm{N}$ & Mínimo & Máximo & Media & Desv. típ. \\
\hline Valoración global & 18 & 3 & 4 & 3,92 &, 251 \\
\hline Entonación & 18 & 3 & 5 & 4,00 &, 333 \\
\hline Actitud & 18 & 3 & 5 & 3,97 &, 424 \\
\hline N válido (según lista) & 18 & & & & \\
\hline
\end{tabular}

Tabla 1. Evaluaciones ISC': Grupo 1

\begin{tabular}{|l|c|c|c|c|c|}
\hline & $\mathrm{N}$ & Mínimo & Máximo & Media & Desv. típ. \\
\hline Valoración global & 18 & 1 & 5 & 3,26 &, 769 \\
\hline Entonación & 18 & 1 & 4 & 2,92 &, 907 \\
\hline Actitud & 18 & 1 & 5 & 3,17 & 1,046 \\
\hline N válido (según lista) & 18 & & & & \\
\hline
\end{tabular}

Tabla 2. Evaluaciones ISM': Grupo 1

\begin{tabular}{|l|c|c|c|c|c|}
\hline & $\mathrm{N}$ & Mínimo & Máximo & Media & Desv. típ. \\
\hline Valoración global & 18 & 4 & 5 & 4,05 &, 229 \\
\hline Entonación & 18 & 4 & 5 & 4,21 &, 419 \\
\hline Actitud & 18 & 4 & 5 & 4,11 &, 315 \\
\hline N válido (según lista) & 18 & & & & \\
\hline
\end{tabular}

Tabla 3. Evaluaciones ISC: Grupo 1

\begin{tabular}{|l|c|c|c|c|c|}
\hline & $\mathrm{N}$ & Mínimo & Máximo & Media & Desv. típ. \\
\hline Valoración global & 18 & 2 & 4 & 2,84 &, 501 \\
\hline Entonación & 18 & 1 & 3 & 2,26 &, 653 \\
\hline Actitud & 18 & 2 & 3 & 2,42 &, 507 \\
\hline N válido (según lista) & $18 \mathrm{c}$ & & & & \\
\hline
\end{tabular}

Tabla 4. Evaluaciones ISM: Grupo 1

Si analizamos las evaluaciones en los tres apartados considerados -valoración global, entonación y actitud- se deducen los siguientes patrones de evaluación según IS (véanse gráficos 3 a 6): 


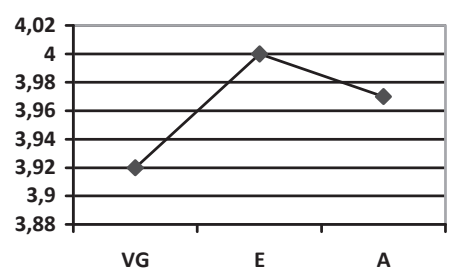

Gráfico 3. Evaluaciones ISC'

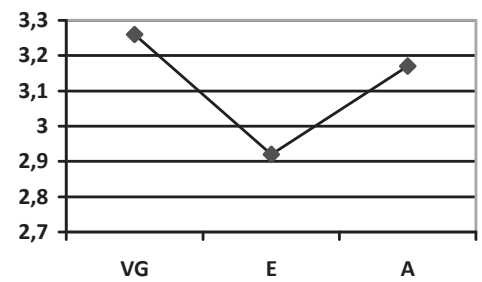

Gráfico 5. Evaluaciones ISM'

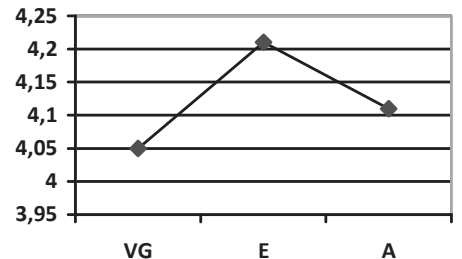

Gráfico 4. Evaluaciones ISC

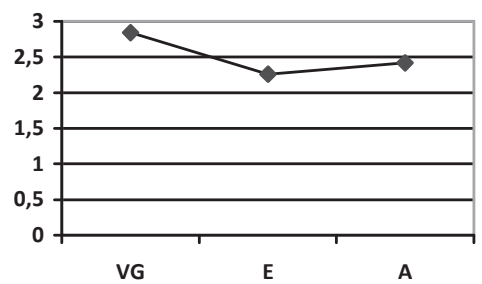

Gráfico 6. Evaluaciones ISM

Si unimos todas las evaluaciones realizadas, con independencia del grado de monotonía de las IS, el resultado ofrece la siguiente curva de evaluación de los ítems considerados (gráfico 7):

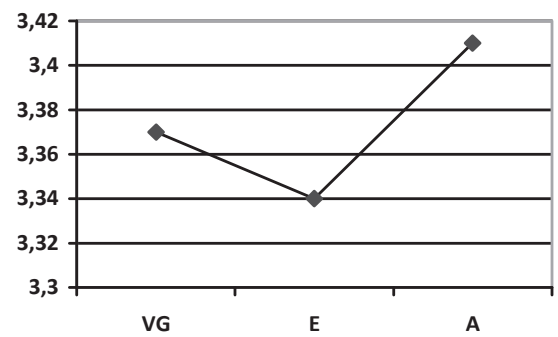

Gráfico 7. Pauta general de evaluación (total IS) del Grupo 1

Por lo que se refiere al Grupo 2, se reflejan a continuación los datos de las valoraciones según los resultados obtenidos para las dos IS monótonas (ISM e ISM') y control (ISC e ISC'). Las pautas evaluadoras resultantes son las siguientes (gráficos 8 y 9): 


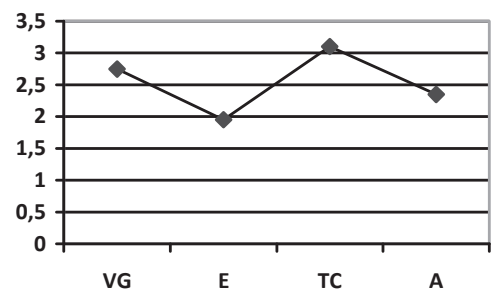

Gráfico 8. Pauta de evaluación: IS monótonas

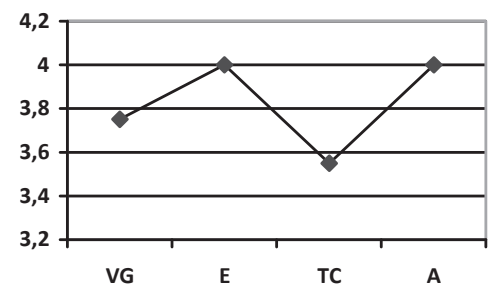

Gráfico 9. Pauta de evaluación: IS no monótonas

A continuación se reflejan las valoraciones conjuntas de las cuatro interpretaciones evaluadas (véase tabla 5 y gráfico 10), con independencia de su grado de monotonía, donde $\mathrm{N}$ equivale al número de evaluaciones emitidas:

\begin{tabular}{|l|c|c|c|c|c|}
\hline & N & Mínimo & Máximo & Media & Desv. típ. \\
\hline Valoración global & 20 & 2 & 5 & 3,25 &, 953 \\
\hline Entonación & 20 & 1 & 5 & 2,98 & 1,240 \\
\hline Transmisión correcta & 20 & 2 & 5 & 3,33 &, 783 \\
\hline Actitud & 20 & 1 & 5 & 3,18 & 1,139 \\
\hline N válido (según lista) & 20 & & & & \\
\hline
\end{tabular}

Tabla 5. Evaluaciones total IS: Grupo 2

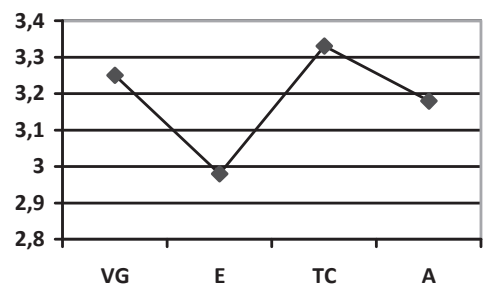

Gráfico 10. Pauta general de evaluación (total IS) del Grupo 2

\subsection{Resultados del Estudio 2}

Tal y como se desprende de la siguiente tabla (véase tabla 6), de las evaluaciones totales de las 30 interpretaciones, el parámetro entonación es el ítem menos valorado por los sujetos. Vemos además que existe una diferencia de prácticamente un punto entre entonación y profesionalidad, así como entre entonación y transmisión correcta. 


\begin{tabular}{|l|c|c|c|c|c|}
\hline & $\mathrm{N}$ & Mínimo & Máximo & Media & Desv. típ. \\
\hline Valoración global & 179 & 1 & 5 & 3,18 &, 919 \\
\hline Transmisión correcta & 180 & 1 & 5 & 3,55 &, 878 \\
\hline Entonación & 178 & 1 & 4 & 2,82 &, 880 \\
\hline Profesionalidad & 173 & 1 & 5 & 3,58 &, 893 \\
\hline Fiabilidad & 171 & 1 & 5 & 3,38 &, 913 \\
\hline N válido (según lista) & 168 & & & & \\
\hline
\end{tabular}

Tabla 6. Evaluaciones total IS: Grupo 3

La pauta general de evaluación respecto a estos mismos parámetros e ítems puede ser reflejada de la siguiente forma (gráfico 11):

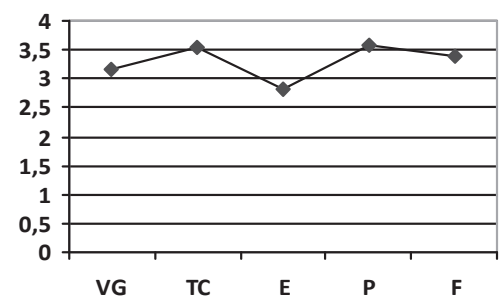

Gráfico 11. Pauta general de evaluación (total IS) del Grupo 3

A fin de analizar las posibles interacciones entre la entonación y los demás ítems considerados, así como una posible pauta evaluadora según las valoraciones más o menos positivas obtenidas por el parámetro entonación, a continuación se exponen, en primer lugar, los resultados referidos a las cinco interpretaciones del corpus que mejor valoración han obtenido en el parámetro entonación -menos monótonas- y, en segundo lugar, las cinco interpretaciones que han obtenido peor valoración en el parámetro entonación -más monótonas-. Se contemplan, como en el apartado anterior, el parámetro transmisión correcta, la valoración global, la profesionalidad y la fiabilidad del intérprete.

En el caso de las cinco interpretaciones que han obtenido valoraciones más positivas en el parámetro entonación, es decir, las consideradas como menos monótonas, este parámetro sigue obteniendo una valoración menor que el resto de ítems considerados (véase tabla 7). Las diferencias van desde el 0,15, en el caso de la valoración global, hasta un máximo de 0,69, en el parámetro transmisión correcta: 


\begin{tabular}{|l|c|c|c|c|c|}
\hline & $\mathrm{N}$ & Mínimo & Máximo & Media & Desv. típ. \\
\hline Valoración global & 30 & 2 & 4 & 3,43 &, 606 \\
\hline Transmisión correcta & 30 & 3 & 5 & 3,97 &, 472 \\
\hline Entonación & 30 & 2 & 4 & 3,28 &, 520 \\
\hline Profesionalidad & 30 & 2 & 5 & 3,85 &, 671 \\
\hline Fiabilidad & 29 & 2 & 5 & 3,69 &, 696 \\
\hline N válido (según lista) & 29 & & & & \\
\hline
\end{tabular}

Tabla 7. Evaluaciones: IS entonación menos monótona

La pauta de evaluación resultante se puede ver en el siguiente gráfico (gráfico 12):

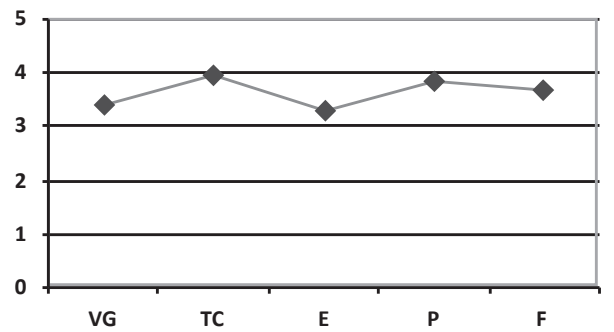

Gráfico 12. Pauta de evaluación: IS entonación menos monótona

En cuanto a los resultados obtenidos por las cinco interpretaciones en las que el parámetro entonación ha sido menos valorado, es decir, las que han sido consideradas más monótonas, podemos comprobar un aumento de como mínimo 0,31 puntos, en el caso de la valoración global, de 0,98 en el caso de la profesionalidad y de 0,86 en el caso de la transmisión correcta, con respecto a la entonación (tabla 8).

\begin{tabular}{|l|c|c|c|c|c|}
\hline & $\mathrm{N}$ & Mínimo & Máximo & Media & Desv. típ. \\
\hline Valoración global & 29 & 1 & 3 & 2,28 &, 621 \\
\hline Transmisión correcta & 30 & 1 & 4 & 2,83 &, 833 \\
\hline Entonación & 30 & 1 & 3 & 1,97 &, 472 \\
\hline Profesionalidad & 30 & 2 & 4 & 2,95 &, 747 \\
\hline Fiabilidad & 30 & 1 & 4 & 2,55 &, 648 \\
\hline N válido (según lista) & 29 & & & & \\
\hline
\end{tabular}

Tabla 8. Evaluaciones: IS entonación más monótona 
La pauta de evaluación resultante se puede ver en el siguiente gráfico (gráfico 13):

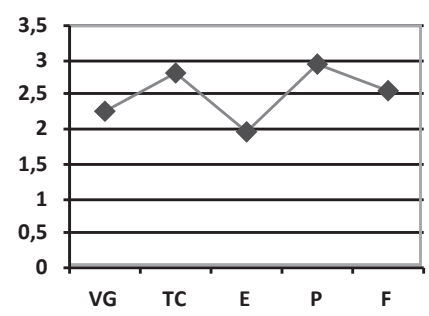

Gráfico 13. Pauta de evaluación: IS entonación más monótona

El contraste entre las pautas evaluadoras vistas en las líneas precedentes, es decir, la pauta general del total de evaluaciones realizadas, con independencia de la valoración emitida en el parámetro entonación, así como las pautas según la valoración menor o mayor de la monotonía de la entonación (E) del intérprete, se ilustra en el siguiente gráfico (gráfico 14):

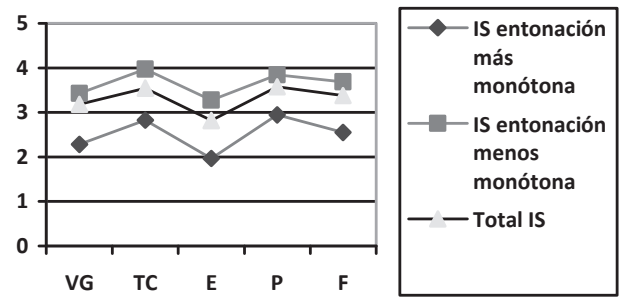

Gráfico 14. Comparación de pautas evaluadoras

\section{Discusión}

Los resultados de los dos estudios han evidenciado pautas de evaluación bastante similares entre sujetos con diferente grado de conocimiento sobre el proceso de la interpretación, si nos referimos a la valoración global de la entonación del conjunto de IS sometidas a evaluación, con independencia de su grado de monotonía. El parámetro entonación se sitúa en la última escala de valoración de los distintos grupos de sujetos y, por tanto, ocupa la franja de valoración baja/media. La entonación es valorada siempre con menor puntuación que el resto de ítems considerados en esta investigación, es decir, que la IS en su conjunto (valoración global), el parámetro transmisión correcta, o la actitud, la profesionalidad y la fiabilidad inferidas del intérprete. 
Estos resultados confirmarían nuestra primera hipótesis e irían en la línea de los resultados obtenidos en distintos trabajos previos sobre evaluación de la calidad en IS (Collados Aís 1998, 2007, 2008, 2010; Pradas Macías 2003). Muy posiblemente la explicación se encuentre en las propias características de la entonación prototípica del intérprete, que ya fue analizada y calificada como sui géneris en diversos trabajos (Shlesinger 1995; Ahrens 2005). Por otra parte, los resultados del Estudio 1, Grupo 1 y Grupo 2, confirmarían los obtenidos en el estudio descrito en § 2.2. En los casos en los que la entonación monótona del intérprete es valorada más negativamente, se produce un aumento en la valoración, respectivamente, de los otros dos y tres ítems considerados. Por el contrario, cuando la entonación del intérprete es valorada más positivamente, es decir, es menos monótona, se produce un descenso en la valoración de los otros ítems. Este resultado no ha sido refrendado en el caso del Grupo 3, especialistas en evaluación de la calidad de la interpretación con un alto grado de conocimiento sobre el proceso de la interpretación. Por tanto, también en este caso, los resultados confirmarían la segunda hipótesis planteada. Una de las posibles explicaciones del efecto descrito que se ha producido en los sujetos con un grado bajo o medio de conocimiento sobre el proceso de la IS podría encontrarse en el modelo previo que estos tengan de la interpretación y que incluiría una cierta monotonía, que incluso se demandaría para determinado tipo de discursos (Collados Aís 2008). Si tenemos en cuenta uno de los resultados más llamativos del estudio que constituye el antedecente inmediato de este (\$ 2.2.), vemos que la actitud del intérprete dejó de definirse por los sujetos de dicho estudio como de neutralidad según aumentaba la melodiosidad de su entonación (al menos en contraste con una entonación monótona). Unido este resultado al papel "fantasma" que demandarían los usuarios del intérprete (Kopczynski 1994), podría deducirse que los sujetos del Estudio 1 deducirían un papel más activo del intérprete en el caso de IS menos monótonas o más melodiosas y, por tanto, menos deseado. El hecho de que este resultado no se haya confirmado en el Estudio 2 tendría su explicación en el hecho de que los sujetos en este caso hayan sido investigadoras sobre la calidad de la interpretación, con un alto grado de conocimiento sobre el proceso de la interpretación, y de que, por tanto, sean más conscientes de que el intérprete, precisamente para prestar una interpretación de calidad, no debe seguir el papel "fantasma" que desearían los usuarios, sino que debe asumir un papel más activo que facilite la comunicación, aunque ello suponga traspasar determinados límites que sobre todo afectarían formalmente a parámetros tan importantes como la transmisión completa o correcta. En el caso de estos sujetos hemos visto cómo las diferencias de evaluación 
de la entonación solamente se situarían en las franjas de valoración, aunque es interesante el hecho de que la diferencia es más acusada en el caso de la peor valoración de la entonación (entonación más monótona) de la interpretación con respecto a la pauta general, que en el caso de la mejor valoración de la entonación (entonación menos monótona) respecto también a la pauta general.

Cabe decir, por último, que existen límites en los que la mayoría de los sujetos se mueven a la hora de valorar una interpretación, tanto en la franja inferior como en la superior, aunque los datos indican que las reservas de los usuarios parecen situarse más en las superiores. Así, los resultados de las valoraciones numéricas de las cuatro interpretaciones analizadas en el Estudio 1 son consistentes con los obtenidos en el Estudio 2. Los sujetos parecen efectivamente seguir unas pautas evaluadoras que tienen unos límites en cuanto a la valoración global, de forma que sus evaluaciones, a pesar de verse influidas por la entonación, no permiten en condiciones de una interpretación ideal plantearse una valoración máxima. Así es difícil que opten por la franja máxima de valoración posiblemente debido a la prevención natural que normalmente podrían tener los receptores, entre otras, la conciencia de la imposibilidad de valorar determinados parámetros, incluso cuando los indicadores puedan permitir inferir que se cumplen.

\section{Conclusiones}

La conclusión más llamativa, con la lógica cautela que imponen muestras reducidas, es que la entonación, globalmente y con independencia de su grado de monotonía, es el parámetro menos valorado por los receptores y no sobrepasa en ningún caso la franja de valoración baja/media. Los receptores parecen percibir que la entonación del intérprete es menos adecuada que otros parámetros de calidad de una IS también sometidos a evaluación, incluido el parámetro por excelencia, la transmisión correcta del sentido, así como la valoración global de la interpretación, la actitud, la profesionalidad y la fiabilidad del intérprete. El hecho de que, a través de sucesivos estudios (cf. Collados Aís 2007), que contemplan tanto interpretaciones auténticas como manipuladas, así como sujetos con diferentes grados de conocimiento sobre el proceso de la interpretación, el resultado sea el mismo nos induce a considerar que, efectivamente la entonación del intérprete, posee características sui géneris (Shlesinger 1994) que llevan a que el usuario valore en último lugar la entonación del intérprete de simultánea.

En cuanto a la pauta de evaluación según el grado de monotonía de la IS, parecen existir diferencias según el grado de conocimiento sobre el proceso 
de la interpretación. En el caso de receptores con un grado bajo o medio de conocimiento, si la entonación de la IS es peor valorada (es considerada más monótona), la valoración global y la transmisión correcta son mejor valoradas, y en el caso de que la entonación sea mejor valorada (sea considerada menos monótona), la valoración global de la interpretación y la transmisión correcta son peor valoradas. En el caso de receptores con un alto grado de conocimiento sobre el proceso de la IS, estos resultados no se confirman. La entonación es valorada, tanto en el caso de que sea menos monótona como más monótona y valorada como tal, peor que el resto de ítems considerados, si bien se producen picos de evaluación más suaves. El receptor con un grado de conocimiento bajo o medio sobre el proceso de la interpretación, a pesar de valorar globalmente mejor la IS y su transmisión correcta en el caso de una valoración más positiva de la entonación, rebaja los límites de las franjas de evaluación en las que se mueve.

Son necesarias nuevas investigaciones que profundicen en los resultados obtenidos, según los cuales se producirían diferentes pautas de evaluación según el grado de monotonía del intérprete de simultánea, así como del grado de conocimiento sobre el proceso de la interpretación. Asimismo, sería necesario ahondar en las causas que provocarían estas diferencias. En este sentido, podría ser interesante analizar si efectivamente a mayor melodiosidad del intérprete, podría existir un límite que se traspasaría, del que el receptor deduciría una actuación demasiado activa del intérprete y que produciría en el usuario cierta desconfianza que se vería reflejada fundamentalmente en sus valoraciones de parámetros de contenido.

En todo caso, los resultados actuales, con la cautela ya señalada, harían necesario un reposicionamiento, tanto en el ámbito profesional como docente, de la entonación del intérprete como parámetro de calidad de la interpretación, habida cuenta de sus múltiples implicaciones sobre su evaluación.

\section{Referencias bibliográficas}

AHRENS, Barbara. (2005) Prosodie beim Simultandolmetschen. Fráncfort del Meno: Peter Lang.

AIIC. (2004) Practical guide for professional conference interpreters. Versión electrónica:<http://aiic.net/page/628/practical-guide-for-professional-conferenceinterpreters/lang/l\#33>

Barranco-Droege, Rafael; Ángela Collados Aís \& José Manuel Pazos Bretaña. (2011) "Intonation." En: Collados Aís, Ángela; Emilia Iglesias Fernández; Esperanza Macarena Pradas Macías \& Elisabeth Stévaux (eds.) 2011. 
Qualitätsparameter beim Simultandolmetschen. Interdisziplinäre Perspektiven. Tubinga: Narr Verlag, pp. 61-92.

Collados Aís, Ángela. (1998) La evaluación de la calidad en interpretación simultánea. La importancia de la comunicación no verbal. Granada: Comares Interlingua.

Collados Aís, Ángela. (2007) "La incidencia del parámetro entonación." En: Collados Aís, Ángela; Esperanza Macarena Pradas Macías; Elisabeth Stévaux \& Olalla García Becerra (eds.) 2007. Evaluación de la calidad en interpretación simultánea: parámetros de incidencia. Granada: Comares, pp. 159-174.

Collados Aís, Ángela. (2008) "Evaluación de la calidad en interpretación simultánea: contrastes de exposición e inferencias emocionales. Evaluación de la evaluación." En: Hansen, Gyde; Andrew Chesterman \& Heidrun GerzymischArbogast (eds.) 2008. Efforts and models in interpreting and translation research: a tribute to Daniel Gile. Ámsterdam/Filadelfia: John Benjamins, pp. 193-214.

Collados Aís, Ángela. (2009) "Marco evaluador de la calidad en interpretación simultánea." En: Bravo Utrera, Sonia \& Rosario García López (eds.) 2009. Estudios de traducción: perspectivas. Zinaida Lvoskaya in memoriam. Fráncfort del Meno: Peter Lang, pp. 145-169.

Collados Aís, Ángela. (2010) "La evaluación de la calidad en interpretación simultánea: pautas evaluadoras según usuarios." En: Rabadán, Rosa; Trinidad Guzmán \& Marisa Fernández (eds.) 2010. Lengua, traducción, recepción. En honor de Julio César Santoyo. León: Universidad de León, pp. 67-90.

Collados Aís, Ángela; Emilia Iglesias Fernández; Esperanza Macarena Pradas Macías \& Elisabeth Stevaux (eds.) 2011. Qualitätsparameter beim Simultandolmetschen. Interdisziplinäre Perspektiven. Tubinga: Narr Verlag.

DÉJEAN LE FÉAL, Karla. (1981) "Lectures et improvisations. Incidences de la forme de l'énonciation sur la traduction simultanée (français-allemand)." Fremdsprachen 1, pp. 29-32.

GARCíA BECERRA, Olalla. (2012) "First Impressions in Interpreting Quality Assessment: The Incidence of Nonverbal Communication." En: Jiménez Ivars, Amparo \& María Jesús Blasco Mayor (eds.) 2012. Interpreting Brian Harris. Recent Developments in Translatology. Fráncfort del Meno: Peter Lang, pp. 173-192.

GARZONE, Giuliana. (2003) "Reliability of quality criteria evaluation in survey research." En: Collados Aís, Ángela; $M^{a}$ Manuela Fernández Sánchez \& Daniel Gile (eds.) 2003. La evaluación de la calidad en interpretación: investigación. Granada: Comares Interlingua, pp. 23-30.

Holub, Elisabeth. (2010) "Does intonation matter? The impact of monotony on listener comprehension." The Interpreter's Newsletter 15, pp. 117-126.

KatZ, Daniel J. (1989) "Pour un enseignement de l'expression orale dans les écoles d'interprètes.” En: Gran, Laura \& John M. Dodds (eds.) 1989. The 
theoretical and practical aspects of teaching conference interpretation. Udine: Campanotto Editore, pp. 217-218.

KOPCZYNKSY, Andrew. (1994) "Quality in conference interpreting: some pragmatic problems.” En: Lambert, Silvie \& Barbara Moser-Mercer (eds.) 1994. Bridging the Gap. Empirical Research in Simultaneous Interpretation. Ámsterdam/ Filadelfia: John Benjamins, pp. 87-99.

KURZ, Ingrid \& Franz Pöchhacker. (1995) "Quality in TV interpreting." TranslatioNouvelles de la FIT- FIT Newsletter 15:3/4, pp. 350-358.

NAFÁ WAASAF, M. Lourdes. (2005) Análisis acústico-discursivo de la entonación en interpretación simultánea inglés británico-español peninsular. Aplicaciones a la didáctica y la investigación de lenguas. Granada: Universidad de Granada. Tesis doctoral.

PRADAS MACÍAS, Esperanza Macarena. (2003) Repercusión del intraparámetro pausas silenciosas en la fluidez: Influencia en las expectativas y en la evaluación de la calidad en interpretación simultánea. Granada. Universidad de Granada. Tesis doctoral inédita.

RUsso, Mariachiara. (2005) "Simultaneous film interpreting and users' feedback." Interpreting 7:1, pp. 1-26.

SHLESINGER, Miriam. (1994) "Intonation in the production and perception of simultaneous interpretation." En: Lambert, Sylvie \& Barbara Moser-Mercer (eds.) 1994. Bridging the gap. Ámsterdam/Filadelfia: Benjamins, pp. 225-236. 


\section{Anexos}

Anexo 1. Cuestionarios de evaluación del Estudio 1

\subsection{Grupo 1}

\section{EVALUACIÓN DE LAS INTERPRETACIONES SIMULTÁNEAS}

Rango de evaluación: 1 (mínima valoración) - 5 (máxima valoración):

\begin{tabular}{|l|l|l|l|}
\hline \multicolumn{1}{|c|}{ IS } & Valoración global & Entonación & Actitud \\
\hline Versión 1 & & & \\
\hline Versión 2 & & & \\
\hline Versión 3 & & & \\
\hline Versión 4 & & & \\
\hline
\end{tabular}

\subsection{Grupo 2}

\section{EVALUACIÓN DE LAS INTERPRETACIONES SIMULTÁNEAS}

Rango de evaluación: 1 (mínima valoración) - 5 (máxima valoración):

\begin{tabular}{|l|l|l|l|l|}
\hline \multicolumn{1}{|c|}{ IS } & $\begin{array}{c}\text { Valoración } \\
\text { global }\end{array}$ & Entonación & $\begin{array}{c}\text { Transmisión } \\
\text { correcta }\end{array}$ & Actitud \\
\hline Versión 1 & & & & \\
\hline Versión 2 & & & & \\
\hline Versión 3 & & & & \\
\hline Versión 4 & & & & \\
\hline
\end{tabular}

Anexo 2. Cuestionario de evaluación Estudio 2: Grupo 3 (ítems considerados en el estudio)

\section{EVALUACIÓN DE LAS INTERPRETACIONES SIMULTÁNEAS}

Identificación de la interpretación:

1. Valoración global (1: pésima; 5: excelente):

\begin{tabular}{|c|}
\hline 1 \\
\hline 2 \\
\hline 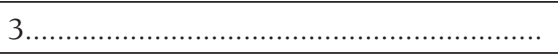 \\
\hline 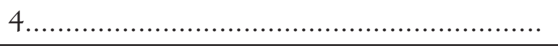 \\
\hline 5 \\
\hline
\end{tabular}


2. Transmisión correcta del sentido del discurso original

(1: transmisión totalmente incorrecta; 5 : transmisión totalmente correcta):

\begin{tabular}{|c|c|}
\hline \multirow[t]{2}{*}{1,1} & \\
\hline & \\
\hline 3 & \\
\hline 4 & \\
\hline & \\
\hline
\end{tabular}

3. Entonación (1: entonación muy monótona; 5: entonación nada monótona):

\begin{tabular}{|c|c|}
\hline \multirow{2}{*}{ 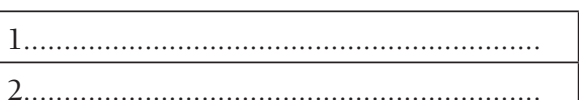 } & \\
\hline & \\
\hline 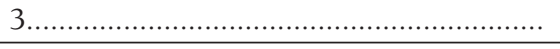 & \\
\hline 4 & \\
\hline 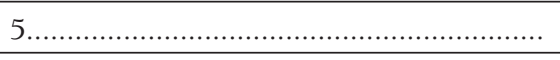 & \\
\hline
\end{tabular}

4. Impresión de profesionalidad (1: nula profesionalidad; 5: alta profesionalidad):

\begin{tabular}{|c|}
\hline $1, \ldots$ \\
\hline 2 \\
\hline 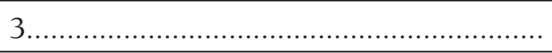 \\
\hline 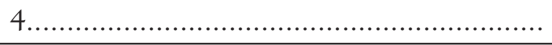 \\
\hline $5 \ldots \ldots \ldots \ldots \ldots \ldots \ldots \ldots \ldots \ldots \ldots \ldots \ldots \ldots \ldots \ldots \ldots \ldots \ldots \ldots \ldots \ldots \ldots \ldots$ \\
\hline
\end{tabular}

5. Impresión de fiabilidad (1: nula fiabilidad; 5: alta fiabilidad):

\begin{tabular}{|c|c|}
\hline \multirow{2}{*}{$\frac{1}{2}$} & \\
\hline & \\
\hline 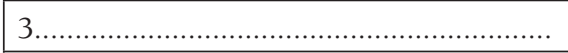 & \\
\hline 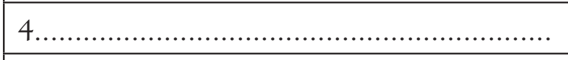 & \\
\hline 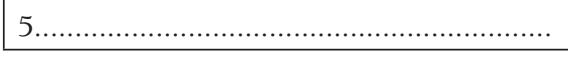 & \\
\hline
\end{tabular}




\section{NOTA BIOGRÁFICA / BIONOTE}

ÁNGELA Collados Aís es diplomada en Traducción e Interpretación (especialidad interpretación), licenciada en Derecho y doctora en Interpretación. Es docente de Interpretación en la Facultad de Traducción e Interpretación de la Universidad de Granada desde 1987, donde imparte interpretación de enlace y de conferencias en la combinación alemán-español. Ha dirigido diversos proyectos de investigación y es la investigadora responsable del grupo de investigación ECIS (Evaluación de la Calidad en Interpretación Simultánea). Sus publicaciones se centran fundamentalmente en el ámbito de la calidad y la docencia de la interpretación.

ÁNGELA COLLADOS Aís holds a diploma in Translation and Interpreting (focus on interpreting), a degree in Law and a $\mathrm{PhD}$ in Interpreting. She has been teaching dialogue and conference interpreting from German into Spanish at the University of Granada, Facultad de Traducción e Interpretación, since 1987. She directed numerous research projects and is the leading researcher of the ECIS (Evaluación de la Calidad en Interpretación Simultánea) research group. Her research work focuses mainly on quality in interpreting and interpreter training. 